\title{
COMMISSION 37: STAR CLUSTERS AND ASSOCIATIONS (AMAS STELLAIRES ET ASSOCIATIONS)
}

\author{
Report of Meetings, 19 and 21 August 1970
}

President: M. Golay.

SECRETARY: G. Lyngå.

\section{A. Administrative Sessions}

1. The Draft Report was approved without corrections.

2. S. Van den Bergh presented to the Commission a copy of the David Dunlap atlas of colourmagnitude diagrams for clusters on the UBV system.

3. J. Jung announced a French data centre for stellar data. Reference was made to Working Group 1 where a more detailed description was to be given.

4. A letter from B. V. Kukarkin informed the Commission about the preparation of the 'General Catalogue of Globular Clusters'. Erroneous data in the literature are corrected and properties of globular clusters are redetermined in a uniform system. Indices of richness (IR) and of metallicity (IM) are also determined.

5. G. A. Alter presented the second edition of the Catalogue of Star Clusters and Associations, which has been published in Budapest by the Publishing House of the Hungarian Academy of Sciences. Alter described the numbering system of clusters according to galactic coordinates, which was accepted at the XIIth General Assembly and now is used for the ordering of the catalogue. A new system for insertion of supplement data is used and the question was put, whether subscribers would be prepared to pay a nominal sum for this service. No objection was raised.

\section{B. Scientific Sessions}

The following papers were given:

Gonzalo Alcaino: 'A UBV Photometric Research on Ten Previously Unstudied Southern Globular Clusters'.

During 1968-70 the 36-in. and 60-in. reflectors at Cerro Tololo Inter-American Observatory have been used to undertake a photometric research on the previously unstudied globular clusters: NGC 1261, NGC 1851, NGC 2808, NGC 4372, NGC 4833, NGC 5286, NGC 6352, NGC 6362, NGC 6541 and NGC 6752.

The following results have been obtained from published photoelectric sequences:

$\begin{array}{lccc}\text { Cluster } & (m-M)_{\mathrm{app}} & E_{(\mathrm{B}-\mathrm{V})} & (m-M)_{\mathbf{0}} \\ \text { NGC 1851 (48 stars) } & 15.15 & 0.24 & 14.43 \\ \text { HGC 2808 (50 stars) } & 15.85 & 0.28 & 15.01 \\ \text { NGC 6362 (61 stars) } & 14.70 & 0.15 & 14.25 \\ \text { NGC 6752 (71 stars) } & 13.50 & 0.00 & 13.50 \\ \text { From calibrated photographic results: } & & & \\ \text { NGC 1261 (206 stars) } & 16.1 & 0.00 & 16.1 \\ \text { NGC 6352 (220 stars) } & 15.40 & 0.44 & 14.10\end{array}$

Complete photoelectric plus photographic results for all ten clusters down to $V \approx 17$ are expected to be completed by 1971 .

O. Maeder: 'Influence of Axial Stellar Rotation on Age Estimates of Open Clusters'.

Individual rotational corrections on the colour-indices of stars depend only on the product 
$v_{\mathrm{R}} \cdot \operatorname{sini}$. Orientation of the axes do not intervene. The absence of these corrections brings about an age overestimate of $60-70 \%$ for Pleiades and $\alpha$ Persei clusters.

G. Janin and P. Bouvier: 'Numerical Experiments on the Disruption of Star Clusters Through Passing Interstellar Clouds'.

The tidal influence on a small stellar cluster of passing interstellar clouds is studied by means of numerical experiments.

The disruption of the cluster occurred over a time scale of $450 \times 10^{6} \mathrm{yr}$ with a cloud concentration of 40 per million cubic parsec.

It is essentially the close encounters which are effective and in particular those of impact parameter smaller than the cloud radius, which had been neglected by Spitzer in his first attempt (1958) to solve this problem.

This paper was illustrated by a film.

P. Pishmish: 'UBV Photometry of the New Open Cluster NGC 2175s, of NGC 2175 and T9'.

UBV magnitudes are determined for stars in the clusters, down to magnitude $V \approx 15^{m} .5$, using photoelectric determinations as standards. The colour-magnitude and color-color diagrams are discussed. The distances are based on the absolute magnitudes, estimated from the spectral types of the brightest stars, and diameters are derived from star-counts. The full text is published in English in the Bol. Obs. Tonantzintla y Tacubaya, 1970 (T9 to be published).

M. Walker: 'Electronographic Photometry of Globular Clusters in the Magellanic Clouds'.

During 1968-69 electrographs of 14 clusters in the Magellanic Clouds were obtained with the spectrocon attached to the $f / 7.5$ focus of the 60 -in. Tololo reflector. Colour-magnitude diagrams for two clusters, Kron 3 and NGC 2209, have been derived and were shown. Photometry to nearly 23rd magnitude is possible by this method, making it possible to use the Magellanic Clouds as a laboratory in which to study the change in the C-M diagram as a function of age and chemical composition. Details of the instrument and technique will appear in the Sept. 1970 issue of Sky and Telescope, and a discussion of the C-M diagram of Kron 3 will appear in the Sept. 1970 issue of the Astrophys. $J$.

R. Wielen: 'On the Life-Times of Galactic Clusters'.

From the observed age distribution of galactic clusters within $1 \mathrm{kpc}$ we deduce that the typical total life-time of a galactic cluster is about $2 \times 10^{8} \mathrm{yr}$. The individual life-times vary between $10^{8}$ and $10^{18} \mathrm{yr}$. The observed life-times are compared with the evaporation times which are found from numerical experiments with star cluster models containing up to 250 stars. The effect of the galactic tidal field is taken into account and this enhances the rate of escape significantly. The agreement between the resulting theoretical life-times and observed values is good. 